\title{
El pluralismo en la televisión digital terrestre en España (2010-2013)
}

\author{
María Dolores MonTERo SÁNCHEZ \\ Universitat Autònoma de Barcelona \\ mariadolores.montero@uab.cat \\ María Jesús DíAZ-GonZÁLEZ \\ Universidade da Coruña \\ m.j.diaz@udc.es \\ Natalia QUINTAS-FroufE \\ Universidade da Coruña \\ n.quintas.froufe@udc.es
}

Recibido: 5 de diciembre de 2014

Aceptado: 8 de mayo de 2015

\section{Resumen}

La implantación de la televisión digital terrestre en España, a partir de abril de 2010 introdujo cambios importantes en el sistema televisivo estatal. El objetivo de este artículo es analizar las principales consecuencias que ha tenido la implantación de la televisión digital terrestre en el pluralismo externo y en el pluralismo interno informativo. Hemos tomado en consideración tres tipos de indicadores de pluralismo externo (propiedad, cuotas de audiencia e inversión publicitaria) desde 2010 hasta 2013 y dos tipos de indicadores del pluralismo interno (índices de correlación entre las cadenas según el orden de relevancia de la información y distribución de los temas por cadenas, en ambos casos aplicados a los titulares) en 2012 y 2013. Se discute la correspondencia entre ambos tipos de pluralismo y se concluye que no existe una relación determinante del pluralismo externo sobre el interno en el período analizado.

Palabras clave: pluralismo externo, pluralismo interno, concentración, comercialización.

\section{Pluralism in Spain's Digital Terrestrial Television (2010-2013)}

\begin{abstract}
The introduction of digital terrestrial television (DTT) in Spain in April 2010 was a major change in the state broadcasting system. The aim of this article is to analyze the main consequences that the introduction of DTT has had on external pluralism and the internal pluralism of the news. External pluralism - the plurality of suppliers - was analyzed using three indicators (ownership, market share and advertising revenues) over the period 2010 - 2013. Internal pluralism - diversity reflected in media content - was analyzed using two indicators (cross-channel correlation of newsworthiness and the distribution of subjects across the channels), which were applied to the headline stories in 2012 and 2013. The article discusses the correlation between the two forms of pluralism and reaches the conclusion that external pluralism has no determining influence on internal pluralism during the period covered.
\end{abstract}

Keywords: external pluralism, internal pluralism, concentration, commercialization.

\section{Referencia normalizada}

MONTERO SÁNCHEZ, María Dolores; DÍAZ-GONZÁLEZ, María Jesús; y QUINTAS-FROUFE, Natalia (2015): "El pluralismo en la televisión digital terrestre en España (2010-2013)". Estudios sobre el Mensaje Periodístico. Especial noviembre "Periodismo e información de calidad", págs.: 209-229. Madrid, Servicio de Publicaciones de la Universidad Complutense.

Sumario: 1. Introducción. 2. Pluralismos mediáticos. 3. Pluralismo externo en el sistema televisivo. 4. Pluralismo interno en la información televisiva. 5. Hipótesis y metodología de investigación. 6. Pluralismo externo: resultados; 6.1. Propiedad; 6.2. Cuotas de audiencia; 6.3. Inversión publicitaria. 7. Pluralismo interno: resultados. 8. Conclusiones. 9. Referencias. 


\section{Introducción}

La discusión sobre el pluralismo en los medios de comunicación es central en la reflexión sobre la calidad de las democracias contemporáneas y el papel de la ciudadanía $^{1}$. La acción de informarse sobre los asuntos públicos, y sobre la controversia sobre ellos, es una de las formas de participación política en las sociedades democráticas (Milbrath y Goel, 1977). El ciudadano tiene derecho a recibir la información necesaria por parte de los representantes políticos y, a la vez, la participación política del ciudadano dependerá de las posibilidades reales de acceder a la información y de procesarla según su conocimiento y su experiencia. La calidad de los medios de comunicación es un elemento importante en la definición de la calidad de la democracia.

La implantación de la televisión digital terrestre en España, a partir de abril de 2010, ha introducido cambios importantes en el sistema televisivo estatal. No obstante, el espíritu optimista inicial, inspirado en las posibilidades de abundancia de canales de la nueva tecnología y en sus hipotéticos efectos de una mayor pluralidad de medios, se ha desvanecido (Bustamante, 2008). En el proceso de implantación de la televisión digital se ha ampliado la oferta de canales, pero también se ha intensificado la concentración de las empresas de medios. Se han podido articular más voces a partir de los nuevos canales, pero la calidad informativa no ha crecido en la misma proporción, tanto en los canales temáticos como en los generalistas.

La política audiovisual de la segunda legislatura del presidente J. L. Rodríguez Zapatero tuvo como resultado la creación de facto de un duopolio en la televisión privada y un debilitamiento de la televisión pública (Bustamante y Corredor, 2012). El gobierno de M. Rajoy, en la misma línea, consolidó el duopolio con la autorización de la fusión de Antena 3 y La Sexta. Respecto a la televisión pública, la modificación del régimen de administración de la Corporación RTVE ha contribuido a una pérdida progresiva de liderazgo de la televisión pública.

El objetivo de este artículo es analizar las principales consecuencias que ha tenido la implantación de la televisión digital terrestre en el pluralismo externo y en el pluralismo interno informativo en el marco del sistema televisivo desde 2010 hasta 2013. El estudio se ha centrado en las cadenas generalistas de cobertura estatal y hemos tomado en consideración tres tipos de indicadores de pluralismo externo (propiedad, cuotas de audiencia e inversión publicitaria) y dos tipos de indicadores del pluralismo interno (índices de correlación entre las cadenas según el orden de relevancia de la información y distribución de los temas por cadenas, en ambos casos aplicados a los titulares).

\section{Pluralismos mediáticos}

La discusión sobre el pluralismo en la información forma parte de la reflexión sobre el papel de los medios de comunicación y su papel en los procesos de creación de la

\footnotetext{
1 Este artículo es resultado del proyecto de investigación "Implantación de la TDT en España e impacto sobre el pluralismo" (The Rollout of Digital Terrestrial Television in Spain and the Impact on Pluralism), financiado por el Ministerio de Ciencia e Innovación (ref. CS201017898/COMU).
} 
opinión pública. Las líneas de argumentación sobre las funciones de los medios están vinculadas a los modelos de democracia (Strömbäck, 2005; Nordestreng, 2006; Karppinen, 2013) y a las formas de participación de la ciudadanía. El pluralismo es un aspecto fundamental para que el ciudadano pueda formar su propia opinión sobre los hechos.

Los numerosos prismas desde los que contemplar el pluralismo hacen que la definición de este concepto sea ardua. Los pluralismos estructural, político, cultural, social, lingüístico, geográfico, de géneros, etc. pueden hacer referencia tanto a la estructura como a los contenidos de los medios. Los diferentes actores políticos, económicos y sociales articulan su visión sobre el pluralismo desde posiciones diferentes y, en muchos casos, contrapuestas.

Una de las visiones más comunes sobre el pluralismo es la de considerar que una mayor competencia en el mercado supone un mayor pluralismo. $\mathrm{O}$, en palabras de Entman, relativas en este caso a la prensa, que "la competencia económica mejora las contribuciones de los diarios al mercado metafórico de las ideas" (Entman, 1989: 93). A juicio del autor, la relación entre competencia económica y calidad de los diarios depende de numerosos factores y estos varían de comunidad a comunidad: "Es difícil predecir una relación consistente entre competencia y contenido de los diarios. En efecto, dado que los mercados de periódicos son tan idiosincrásicos, son igualmente plausibles las predicciones directamente contradictorias sobre los efectos de la competencia" (Ibíd.: 93). Lo que más influiría en la calidad de los diarios, según Entman, serían las prioridades de la empresa, los intereses de la audiencia y los anunciantes, y no la mayor o menor competencia.

Este artículo parte de la diferenciación entre pluralismo externo e interno. Ambos conceptos fueron propuestos por el Consejo de Europa, a partir del Informe del Comité de Expertos sobre Concentración de medios y Pluralismo (1994), entregado a la IV Conferencia ministerial sobre política de medios de comunicación de masas en una sociedad democrática. Se considera que el pluralismo externo se manifiesta en la diversidad de medios, empresas o grupos de interés, que expresan una opinión particular. Desde este punto de vista, la diversidad emerge de los grupos políticos, económicos y sociales hegemónicos en la sociedad que tienden a definir la agenda de temas de actualidad. El pluralismo externo hace referencia al pluralismo estructural de los medios, la propiedad, el grado de competencia entre las empresas y la regulación de los medios. Por lo general, parte de la premisa de que estos aspectos son determinantes, con mayor o menor intensidad, en la oferta de programas y contenidos del medio.

El pluralismo interno se expresa a través de la diversidad de puntos de vista y opiniones en el seno de un medio. Hace referencia a las distintas posiciones frente a una situación o en un debate de ideas. El pluralismo interno da lugar al acceso de un mayor número de grupos al debate político $\mathrm{y}$, en consecuencia, la información tiende a ser más equilibrada. En la medida en que incorpora puntos de vista de un abanico más amplio de grupos sociales procura una discusión más horizontal en la que se pueden sentir implicados un mayor número de ciudadanos. El pluralismo interno remite principalmente a los contenidos y a la diversidad de grupos políticos, económicos y sociales que tienen acceso a los medios. 
La preocupación por la contribución de los medios al funcionamiento de la democracia se ha reflejado en los esfuerzos por desarrollar mecanismos de observación sobre los pluralismos mediáticos (Just, 2009; Iosifidis, 2010). La Comisión Europea está implementando, desde 2009, un sistema de Monitoreo del Pluralismo de los Medios en los estados miembros de la Unión Europea para identificar los posibles riesgos (K. U. Leuven-ICRI et al., 2009; Vike-Freiberga et al., 2013). En el Independent Study on Indicators for Media Pluralism, realizado por K. U. Leuven et al. (2009) a petición de la Comisión Europea, se adoptó una definición de pluralismo que integraba el estudio del pluralismo externo e interno para detectar las situaciones de riesgo de los medios de comunicación en las democracias maduras. Desde su punto de vista, el pluralismo se entiende como el ámbito de "una amplia gama de valores culturales, políticos y sociales, opiniones, información e intereses que se expresa a través de los medios" (K. U. Leuven et al., 2009: 5). El sistema de monitoreo propuesto en este estudio parte de la combinación de indicadores cuantitativos y cualitativos para analizar, en las democracias maduras, los diferentes aspectos del pluralismo en los medios.

En la línea de argumentación de Entman (1989) cabe preguntarse cuál es la relación entre ambos tipos de pluralismo, externo e interno, y cómo se podría valorar el grado de pluralismo existente en un sistema de medios. Así, Iosifidis (2010) considera que se deberían combinar varios tipos de métodos, para establecer el impacto de los medios sobre la audiencia, basados en las medidas del poder económico en los mercados y en las medidas del pluralismo y la diversidad. Desde una perspectiva más crítica, Karppinen pone de manifiesto la contradicción de que "mientras que unos piensan que la abundancia de medios implica una indudable dispersión de poder, otros consideran que nos puede llevar muy lejos del ideal de una esfera plural libre donde las diferentes perspectivas entran en juego" (Karppinen, 2013: 4).

\section{Pluralismo externo en el sistema televisivo}

La legislación española sobre el audiovisual delimita el marco regulador del pluralismo. La Ley 17/2006, de 5 de junio, de la radio y la televisión de titularidad estatal, se fundamenta en el artículo 20.3 de la Constitución. En dicho artículo, se explicita que los medios de comunicación social dependientes del Estado o de cualquier ente público deben garantizar el acceso a los grupos sociales y políticos. Y, continúa, deben garantizar el acceso respetando el pluralismo de la sociedad y de las diversas lenguas de España. La actividad de los medios públicos debe regirse por el criterio de servicio público y promocionar el pluralismo para garantizar el acceso a los grupos sociales y políticos significativos.

La Ley General de la Comunicación Audiovisual (ley 7/2010, de 31 de marzo) tiene por objetivo regular el conjunto del sector audiovisual. Dicha Ley especifica también el "derecho a recibir una comunicación audiovisual plural" en los siguientes términos:

Todas las personas tienen el derecho a que la comunicación audiovisual se preste a través de una pluralidad de medios, tanto públicos, comerciales como comunitarios que reflejen el pluralismo ideológico, político y cultural de la sociedad. Además, todas las 
personas tienen el derecho a que la comunicación audiovisual se preste a través de una diversidad de fuentes y de contenidos y a la existencia de diferentes ámbitos de cobertura, acordes con la organización territorial del Estado. Esta prestación plural debe asegurar una comunicación audiovisual cuya programación incluya distintos géneros y atienda a los diversos intereses de la sociedad, especialmente cuando se realice a través de prestadores de titularidad pública (Ley General de la Comunicación Audiovisual [ley 7/2010, de 31 de marzo], art. 4).

Además de este principio que se incluye como normativa básica de la comunicación audiovisual, esta Ley dedica específicamente el artículo 36 al pluralismo en el mercado audiovisual televisivo. El pluralismo se concibe sólo como pluralismo externo, y se piensa que de este pluralismo externo derivará el pluralismo informativo. Lo que se pretende es permitir la concentración de la propiedad de las empresas de TDT; pero con unos límites que se refieren a la concentración de las audiencias, la concentración de capacidad de emisión y el número mínimo de empresas privadas que tienen que estar presentes en el mercado.

Las personas físicas y jurídicas pueden ser titulares simultáneamente de participaciones sociales o derechos de voto en diferentes prestadores del servicio de comunicación audiovisual televisiva.

[esto no se autorizará] cuando la audiencia media del conjunto de los canales de los prestadores de ámbito estatal considerados supere el $27 \%$ de la audiencia total durante los doce meses consecutivos anteriores a la adquisición.

[ni] cuando los prestadores del servicio de comunicación audiovisual de ámbito estatal acumulen derechos de uso sobre el dominio público radioeléctrico superiores, en su conjunto, a la capacidad técnica correspondiente a dos canales múltiplex.

[y tampoco] cuando ello suponga impedir la existencia de, al menos, tres prestadores privados distintos del servicio de comunicación audiovisual televisiva en el ámbito estatal, asegurándose el respeto al pluralismo informativo (Ley 7/2010, de 31 de marzo, art. 36).

Después de la política audiovisual de la primera legislatura del gobierno de J. L. Rodríguez Zapatero (Zallo, 2010), la aplicación de la Ley General de la Comunicación Audiovisual fue paradójica en relación a los avances obtenidos hasta entonces. La política audiovisual del gobierno de M. Rajoy ha seguido una línea continuista en los siguientes aspectos: la consolidación del duopolio formado por Mediaset y Atresmedia (García-Santamaría, 2013; Díaz-González y Labio Bernal, 2012; Díaz-González y Quintas Froufe, 2013); la obstaculización del desarrollo de los medios comunitarios; las dificultades de financiación de la televisión pública y la no creación de un organismo regulador de ámbito estatal, el Consejo Estatal de Medios Audiovisuales, previsto en la ley de 2010. Las funciones que debía tener este Consejo las asumió en octubre de 2013 la Comisión Nacional de los Mercados y la Competencia $(\mathrm{CNMC})^{2}$.

2 Esta Comisión se constituye por la Ley 3/2013, de 4 de junio, de Creación de la Comisión Nacional de los Mercados y la Competencia. 
Después del mapa televisivo que se estableció tras el apagón analógico, la situación de la TDT de cobertura nacional que se fue configurando con la Ley 7/2010, General de la Comunicación Audiovisual, y con las decisiones de los gobiernos de J. L. Rodríguez Zapatero y M. Rajoy a las que nos hemos referido, iba a verse profundamente afectada por unos hechos poco previsibles.

En diciembre de 2013, la Sala de lo Contencioso-Administrativo del Tribunal Supremo dictó un auto que ordenaba la ejecución de su sentencia de 27 de noviembre de 2012, por la que nueve canales de TDT de cobertura nacional debían dejar de emitir porque su atribución era nula y, por tanto, carecían de título para desarrollar su actividad. El motivo de que su atribución fuese nula derivaba de que fueron asignados directamente por el gobierno (segunda legislatura de J. L. Rodríguez Zapatero) sin que se convocase concurso público, según lo establecido en la ley (Ley 7/2010, de 31 de marzo, General de la Comunicación Audiovisual).

Cuando se hizo pública la sentencia de noviembre de 2012, el Gobierno ya había cambiado (legislatura de M. Rajoy). Éste decidió que ejecutaría lo que le ordenaba el Tribunal Supremo, pero estableció que los canales siguieran emitiendo hasta que culminase el proceso de liberación del dividendo digital. Y aquí, precisamente, es donde incidía el auto al que nos estamos refiriendo: el Supremo anuló esta espera por el dividendo digital; los canales debían dejar de emitir (Comunicación Poder Judicial, 2013).

Como era de esperar, los operadores de televisión afectados recurrieron este auto del Tribunal Supremo ${ }^{3}$; pero todos los recursos fueron desestimados en un nuevo auto con fecha 13 de febrero de 2014 (Tribunal Supremo. Sala de lo Contencioso, 2014a). A partir de esta fecha, todos los afectados sabían que empezaba la cuenta atrás para que nueve canales de TDT cerraran. El 25 de marzo de 2014, el Tribunal Supremo intervino de nuevo para requerir información al Ministerio de Industria, Energía y Turismo sobre quién sería el responsable del cumplimiento de la sentencia y para indicarle que debía decretar el cese de las emisiones de los canales de TDT afectados. Además, señaló y recordó que el plazo para que se produjera este cese terminaba el 6 de mayo de 2014 (Tribunal Supremo. Sala de lo Contencioso, 2014b) . $^{4}$

En abril de 2014, los cuatro operadores (Atresmedia, Mediaset, Net TV y Veo Televisión) recibieron un requerimiento desde la Secretaría de Estado de Telecomunicaciones y para la Sociedad de la Información (SETSI) para que dejaran de emitir los canales afectados. A pesar de las protestas hechas públicas por la UTECA y de las acciones de movilización emprendidas por agentes que se sienten afectados (las Productoras Asociadas de Televisión de España y la Federación de Asociaciones de Consumidores y Usuarios de los Medios, entre otras), el cierre de los canales se llevó a cabo en la fecha prevista.

3 La UTECA, Net TV, Veo Televisión, Atresmedia y Mediaset interpusieron recursos de reposición contra el auto del Tribunal Supremo de 18 de diciembre de 2013.

${ }^{4}$ Como señala el Auto, el plazo termina el 6 de mayo de 2014 porque fue el 6 de marzo de 2014 cuando entró en la Subdirección General de Recursos del Ministerio de Industria, Energía y Turismo el requerimiento relativo al cumplimento del Auto de ejecución, y el artículo 104.2 de la Ley de Jurisdicción establece un plazo de dos meses entre la comunicación y el cumplimiento. 
Como hemos señalado antes, la intención inicial del gobierno de M. Rajoy era hacer coincidir el apagado de estos canales con la liberación del dividendo digital. España está comprometida a que sus operadores de televisión dejen libre el dividendo digital ${ }^{5}$ para enero de 2015; este proceso afecta directamente a las televisiones privadas con cobertura nacional porque la mayoría de sus canales emiten actualmente en esas frecuencias que hay que liberar. Además, este proceso les va a suponer un coste añadido porque tendrán que emitir simultáneamente en dos frecuencias distintas durante el tiempo que dure el cambio, con el riesgo de perder audiencia en la mudanza de una frecuencia a otra.

\section{Pluralismo interno en la información televisiva}

En el acuerdo del Consejo de Ministros de agosto de 2012 por el que se autoriza la operación de concentración económica Antena3/La Sexta, el gobierno de M. Rajoy definió el pluralismo informativo. Uno de los objetivos por los que el gobierno justificó su intervención, tras las condiciones establecidas por la Comisión Nacional de la Competencia, estaba relacionado con la garantía del pluralismo informativo, la concurrencia libre de ideas y corrientes de opinión que diferencia unos concesionarios de otros. Además, dicho acuerdo explicitaba que el Consejo de Europa y la normativa europea (Directiva 2010/13/UE del Parlamento europeo y del Consejo, del 10 de marzo de 2010, Directiva de servicios de comunicación audiovisual) entendían el pluralismo como "una diversidad de medios independientes y autónomos, así como de contenidos, a disposición del público". Obviamente, una interpretación del pluralismo basada principalmente en el pluralismo externo.

La fragmentación de las audiencias a la que ha conducido la TDT ha influido indirectamente sobre los informativos. En las cadenas generalistas la competencia entre los programas de información diaria se ha reflejado en una fuerte acentuación de la rivalidad por las audiencias. A finales de 2013 los informativos de noche de Telecinco ya habían ocupado la primera posición, por delante de La 1, y, en tercer lugar, los de Antena 3. En la televisión pública, la pérdida progresiva de audiencia en los informativos de TVE1 se ha unido, además, a una merma importante de credibilidad que se ha visto reflejada en varias ocasiones en cuestionamientos públicos sobre el enfoque de las noticias.

La tendencia a la comercialización de la información tiende a modificar el papel de los medios y su relación con los actores políticos y sociales. El impacto de la comercialización afecta a aspectos estructurales del sistema de medios y a su relación con la política. Desde la perspectiva de Hallin y Mancini (2004) las consecuencias de la

5 Se denomina "dividendo digital" a la banda de frecuencias del espectro radioeléctrico (790862 MHz: canales 61 a 69) que se están utilizando para dar servicio de TDT y van a pasar a dar servicio de telefonía móvil de cuarta generación 4G. Se considera que este proceso tendrá una repercusión muy importante en la economía nacional. Para ampliar información se puede consultar el Plan Marco de actuaciones para la liberación del dividendo digital 20122014. Recuperado: 9 de mayo de 2013 de: http://www.minetur.gob.es/telecomunicaciones/esES/Novedades/Documents/Plan_Marco_Dividendo_Digital.pdf 
comercialización se expresan en los siguientes aspectos: a) modificación de la función social del periodismo, en la medida en que aleja a los medios de la difusión de ideas y la creación de consenso; b) repercusión en los procesos de comunicación política, puesto que desarrollan dinámicas de creación de audiencias a las que los partidos políticos necesitan adecuarse (si desean establecer un flujo comunicativo constante con la ciudadanía); c) tendencia a centrarse en la perspectiva del «ciudadano de a pie»; y d) un cambio en el equilibrio entre las instituciones políticas y los medios de comunicación, de manera que éstos tienden a establecer la agenda política.

El modelo televisivo español es un modelo híbrido, con diferentes gradaciones de pluralismo externo e interno según cada cadena. Se podrían distinguir tres ejes principales de selección y producción de las noticias en las redacciones informativas. Así, las cadenas que acentúan el carácter comercial de las noticias serían Telecinco, Cuatro y Antena 3; las que se dirigen a nichos de audiencia orientados ideológicamente serían La Sexta e Intereconomía; y, finalmente, TVE se caracterizaría por ser la cadena con un mayor pluralismo interno (Humanes et al., 2013).

En los últimos años han sido varias las investigaciones que se han centrado en las consecuencias del proceso de comercialización en la televisión y, en particular, en la información televisiva (López y Cuenca, 2005; León, 2006; Miguel y Pozas, 2009) y todas ellas coinciden en que produce un claro efecto de homogeneización. Aún cuando la televisión digital terrestre en España ha introducido canales dirigidos a nichos de audiencia polarizados, en la práctica los procesos de comercialización y de polarización ideológica de la información pueden tener resultados similares en lo que respecta al uso de las fuentes de información (Montero y Humanes, 2013).

\section{Hipótesis y metodología de investigación}

El objetivo de este artículo es analizar las principales consecuencias que ha tenido la implantación de la televisión digital terrestre en el pluralismo externo y en el pluralismo interno informativo en el marco del sistema televisivo desde 2010 hasta 2013. Se han analizado las cadenas generalistas de cobertura estatal: Telecinco, Antena 3, La 1, La Sexta, Cuatro e Intereconomía. En este conjunto de cadenas se descartó inicialmente 13 TV porque, en el momento de inicio de la investigación, en 2011, su cuota de audiencia $(0,4 \%)$ era muy baja (Barlovento, 2011).

Las hipótesis de trabajo han sido las siguientes:

H1: El pluralismo externo se ha reducido debido al proceso de concentración de los grupos mediáticos y a la evolución de la inversión publicitaria.

H2: El pluralismo interno informativo se ha reducido debido a la lógica de comercialización de la información diaria.

H3: La relación entre pluralismo externo e interno está vinculada a las formas de gestión y estrategias de las empresas mediáticas.

En el análisis del pluralismo externo hemos considerado tres tipos de indicadores: propiedad, cuotas de audiencia e inversión publicitaria, desde 2010 hasta 2013. El método de trabajo se ha basado en la consulta documental, comparación de los datos y entrevistas con los agentes afectados por el proceso de implantación de la TDT.

En el análisis del pluralismo interno informativo hemos tenido en cuenta dos indicadores: índices de correlación entre las cadenas según el orden de relevancia de la 
información y distribución de los temas por cadenas, en ambos casos aplicados a los titulares. La metodología ha sido el análisis de contenido de los titulares de los informativos de noche de las cadenas de televisión generalistas de cobertura estatal anteriormente citadas. Dado que esta investigación se inserta en un proyecto más amplio, algunos de los resultados del análisis de contenido cuantitativo ya se han divulgado en distintas publicaciones (Humanes et al., 2013; Montero y Humanes, 2013). En este artículo presentamos los resultados del análisis de contenido de los titulares con el objetivo de avanzar en la interpretación de resultados.

Los titulares son un indicador de la relevancia de la información que los distintos medios dan a las noticias. La sucesión de titulares propone un esquema de comprensión de la actualidad a partir de los criterios establecidos por el programa, e indirectamente por la empresa, en la producción de noticias. Los titulares expresan el punto de vista del programa y también reflejan los recursos argumentativos e icónicos para atraer la atención del público.

El período de análisis ha sido de cuatro semanas, dos de 2012 y dos de 2013. La dos semanas de 2012 se seleccionaron de manera aleatoria ( 23 al 29 de enero y 13 al 19 de febrero) y se eligieron las mismas fechas para el año 2013. En estas cuatro semanas, en los informativos analizados (Informativo 21 horas, Telecinco; Noticias 2, Antena 3; Telediario 21 horas, La 1; Noticias 2, La Sexta; Noticias 20 horas, Cuatro; y El Telediario, Intereconomía) se obtuvieron un total de 1.267 titulares. Entre este conjunto de titulares prescindimos de los que sólo aparecían en un medio en un mismo día. Así, el número de titulares descartados en 2012 fueron 174 y en 2013 alcanzaron los 166. Esto nos garantizó un mínimo de interés general, o noticiabilidad, del titular y permitió que la comparativa entre los criterios de selección y relevancia de los medios se hiciera en un cuerpo más homogéneo de eventos significativos. Después de este proceso de selección el corpus resultante fue de 479 titulares en 2012 y 448 en 2013. Es decir, un total de 927 titulares.

Para analizar el grado de pluralismo en los programas escogidos y corroborar, o no, la $\mathrm{H} 2$ nos hemos centrado en los temas de la información y en el orden de relevancia de estos temas expresados en los titulares. Consideramos que la lógica de comercialización de la información diaria puede conducir a una homogeneización en la selección de los temas más importantes o bien a una dispersión, en el caso de las noticias blandas más tendentes al entretenimiento.

Para comparar de un modo objetivo cómo cada medio destaca los eventos más noticiables, hemos sintetizado una variable de relevancia que viene cuantificada por dos factores: 1) el número de orden que ocupa en los titulares, que indica el posicionamiento del programa respecto a la relevancia de la noticia; y 2) el número de piezas presentadas en los titulares en un día determinado. Esta variable de relevancia se definiría con la siguiente fórmula: $\mathrm{R}=\mathrm{f}(\mathrm{X}, \mathrm{Y}), \mathrm{Rmax}=100, \mathrm{Rmin}=\varnothing$. La función de relevancia asigna un valor entre 100 y $\varnothing$, según el orden que ocupa la noticia $(X)$ y el número de noticias destacadas en los titulares $(\mathrm{Y}) . \mathrm{R}=\varnothing$ si la noticia no aparece en los titulares. Así pues $\mathrm{R}=(100 / \mathrm{Y})(\mathrm{Y}-\mathrm{X}+1)$, donde $\mathrm{X}=$ número de orden del titular e $\mathrm{Y}=$ total de titulares destacados. 


\section{Pluralismo externo: resultados}

Como ya hemos señalado, para el estudio del pluralismo externo se han considerado tres tipos de indicadores: propiedad, cuotas de audiencia e inversión publicitaria, desde 2010 hasta 2013.

La selección de estos indicadores se basa en las recomendaciones del Independent Study on Indicators for Media Pluralism. Towards a Risk-Based Approach, realizado por K. U. Leuven et al., por encargo de la Comisión Europea. Este informe señala la propiedad de los medios y su control como una dimensión del pluralismo mediático donde pueden presentarse riesgos o amenazas; y, para evaluar la presencia de estos riesgos propone entre otros los indicadores a los que nos estamos refiriendo $(\mathrm{K}$. U. Leuven-ICRI et al., 2009: 11-14 y 75-76).

Además, el estudio de la propiedad (en el que queda constancia del número de empresas presentes en el mercado y del número de múltiplex que poseen) y de las cuotas de audiencia es coherente con lo que hemos señalado que establece la Ley General de la Comunicación Audiovisual sobre el pluralismo en el mercado audiovisual televisivo.

\subsection{Propiedad}

Entre los años 2010 y 2013, el número de compañías que estaban en posesión de una licencia para emitir TDT de cobertura estatal en España se redujo de siete a cinco. En marzo de 2010 (fecha del apagón analógico en España), estaban funcionando Televisión Española (TVE), Gestevisión Telecinco (en adelante Mediaset España que es su denominación desde abril de 2011), Antena 3 de Televisión (en adelante Atresmedia que es su denominación desde abril de 2013), Sogecable, Gestora de Inversiones Audiovisuales La Sexta, Net TV y Veo TV. Al finalizar 2013, las compañías operativas eran: TVE, Mediaset España, Atresmedia, Net TV y Veo TV.

La primera operación de concentración entre operadores de televisión se cerró en diciembre de 2010, cuando Mediaset España firmó la compra de la licencia de TDT de cobertura estatal de Sogecable (grupo Prisa) (Díaz González y Labio Bernal, 2012). Y la segunda operación terminó en octubre de 2012, fecha en la que Atresmedia firmó la absorción del cien por cien de Gestora de Inversiones Audiovisuales La Sexta (en adelante La Sexta). Por tanto, a finales del año 2013 se cumplía un año de la existencia de un sistema televisivo (de cobertura nacional) en el que conviven una empresa pública (TVE) y cuatro empresas privadas (Mediaset España, Atresmedia, Net TV y Veo TV).

En el mismo período (2010-2013) el número de canales emitidos con cobertura nacional pasó de 20 a 34, lo que supuso un incremento del $70 \%$ en la capacidad de emisión de contenidos televisivos; es decir, en el número de horas de televisión ofertadas a los ciudadanos.

Además, el número de empresas de televisión -propietarias de licencias- que realmente tomaban decisiones sobre los contenidos emitidos había quedado casi reducido a tres (TVE, Mediaset España y Atresmedia), porque Veo TV no producía ninguno de los canales que emitía y los socios de Net TV solo producían dos (Intereconomía y Disney Channel) de los cuatro canales que emitían. A todo esto hay que añadir que el 58,8 \% de la capacidad de emisión en esta cobertura estaba concentrada 
en dos operadores, Mediaset España y Atresmedia, ya que estos poseían 20 canales de los 34 que existían.

Esta situación con la que finalizó el año 2013 se vio modificada en los primeros meses de 2014 por el proceso de aplicación de la sentencia del Tribunal Supremo sobre el cierre de canales de TDT, al que antes hemos hecho referencia. El 6 de mayo de 2014, al dejar de emitir 9 canales, el total de canales de TDT de cobertura nacional pasó de 34 a 25. Atresmedia tiene siete y Mediaset España tiene ocho; como consecuencia, entre ambas concentran el $60 \%$ de la capacidad de emisión, aún más que a finales de 2013. Los dueños de Net TV solo producen un canal (Disney Channel) de los dos que la empresa emite, y Veo TV sigue sin producir ninguno. Por tanto, continúa la situación de que solo tres empresas gestionan directamente los contenidos que emiten.

Con todo, los que eran propietarios de las televisiones en 2010 siguen presentes en las transformadas compañías que existen a mediados de 2014; su participación accionarial y su presencia en la gestión ha cambiado pero ninguno había dejado el negocio de la televisión, hasta ese momento.

El grupo Prisa vendió su televisión en abierto pero era propietario (en un 13,65 \%) de Mediaset España. Asimismo, los propietarios de La Sexta se deshicieron también de su licencia, pero son socios de Atresmedia (en un 6,49\%). En este caso, es preciso aclarar que los propietarios de La Sexta en el año 2010 eran GAMP Audiovisual, Gala Desarrollos Comerciales y Televisa (el grupo televisivo mexicano). Al decidirse la venta de esta empresa a Atresmedia, el grupo Televisa decidió que no continuaría participando en el negocio de televisión en abierto en España; por eso, a cambio del $40,52 \%$ que tenía en La Sexta, se acordó que recibiría un 14,5\% del capital de Imagina Media, que sí iba a ser nuevo socio en Atresmedia. Como podemos ver, Prisa, GAMP Audiovisual, Imagina Media o Televisa han renunciado a la gestión de su licencias de TDT pero se mantienen como socios en el capital de otras compañías de televisión, que están siendo rentables a pesar de la crisis económica que afecta al sector.

\begin{tabular}{|c|c|c|c|}
\hline \multicolumn{2}{|c|}{2010} & \multicolumn{2}{|c|}{2013} \\
\hline $\begin{array}{l}\text { Empresas con licencia de } \\
\text { TDT de cobertura nacional }\end{array}$ & Accionistas & $\begin{array}{l}\text { Empresas con licencia de } \\
\text { TDT de cobertura nacional }\end{array}$ & Accionistas \\
\hline Antena 3 de Televisión, S.A. & $\begin{array}{l}\text { Planeta-De Agostini } \\
(44,58 \%) \\
\text { RTL (Bertelsmann) } \\
(20,49 \%) \\
\text { Autocartera }(5 \%) \\
\text { Bolsa }(29,93 \%)\end{array}$ & $\begin{array}{l}\text { Atresmedia Corporación de } \\
\text { Medios de Comunicación, S.A. }\end{array}$ & $\begin{array}{l}\text { Planeta-De Agostini } \\
(41,70 \%) \\
\text { RTL (Bertelsmann) } \\
(19,17 \%) \\
\text { Imagina Media } \\
\text { Audiovisual, S.A. } \\
\text { (6,49\%) } \\
\text { Autocartera }(7,01 \%) \\
\text { Bolsa }(25,63 \%)\end{array}$ \\
\hline Gestevisión Telecinco, S.A. & $\begin{array}{l}\text { Mediaset Investimenti SpA } \\
(41,55 \%) \\
\text { Prisa Televisión, S.A. } \\
(17,34 \%) \\
\text { Tweedy Browne Company } \\
\text { Llc }(4,41 \%) \\
\text { Blackrock Investment } \\
\text { Management (UK) Ltd } \\
\text { (3,02\%) } \\
\text { FMR Lle (EEUU) }(2,49 \%) \\
\text { Autocartera }(1,58 \%) \\
\text { Bolsa }(29,61 \%)\end{array}$ & $\begin{array}{l}\text { Mediaset España } \\
\text { Comunicación, S.A. }\end{array}$ & $\begin{array}{l}\text { Mediaset Investimenti } \\
\text { SpA }(41,55 \%) \\
\text { Prisa Televisión, S.A. } \\
(17,34 \%) \\
\text { Tweedy Browne } \\
\text { Company Llc }(4,41 \%) \\
\text { Autocartera }(1,59 \%) \\
\text { Bolsa }(35,11 \%)\end{array}$ \\
\hline
\end{tabular}




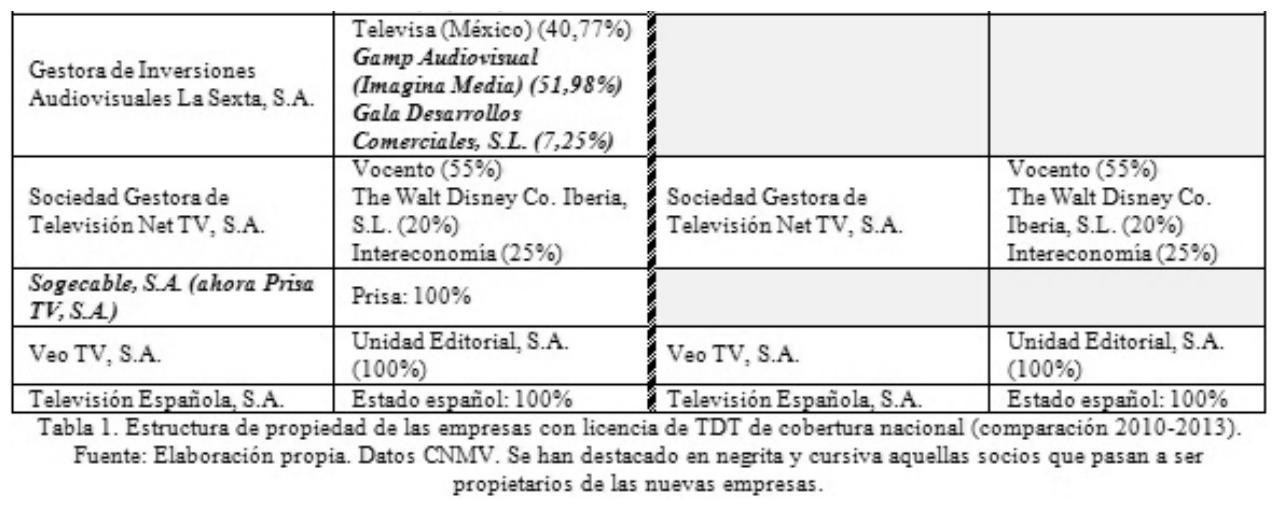

\subsection{Cuotas de audiencia}

Con la implantación de la TDT, y el consiguiente apagón analógico, se inició el proceso de fragmentación de las audiencias en España. La multiplicación de la oferta televisiva, fruto del incremento del número de canales, marcó una nueva etapa en el flujo de las audiencias.

Para valorar la evolución de las audiencias televisivas durante el período estudiado convendría señalar dos cambios que influyeron en su trayectoria:

Por una parte, el aumento del consumo televisivo de forma destacable. En el año 2012 se alcanzó el máximo histórico de minutos consumidos por persona y día (246), cifra que descendió en 2013 (244 minutos por persona y día) pero que logró ser el segundo año de mayor consumo televisivo en España.

Por otra parte, la fusión de Cuatro y Telecinco y la absorción de La Sexta por parte de Atresmedia configuraron un nuevo escenario televisivo que cambió significativamente el flujo de las audiencias. El dominio de ambos grupos puede verse reflejado en la cuota de pantalla agregada recogida en la tabla 2.

\begin{tabular}{|l|l|l|l|l|}
\cline { 2 - 5 } \multicolumn{1}{c|}{} & $\mathbf{2 0 1 0}$ & $\mathbf{2 0 1 1}$ & $\mathbf{2 0 1 2}$ & $\mathbf{2 0 1 3}$ \\
\hline Grupo Atresmedia & $15,8 \%$ & $17,1 \%$ & $25,8 \%$ & $28,8 \%$ \\
\hline Grupo Mediaset España & $17,7 \%$ & $26,4 \%$ & $28,1 \%$ & $29 \%$ \\
\hline Crtve & $24,1 \%$ & $22,3 \%$ & $18,9 \%$ & $16,7 \%$ \\
\hline La Sexta & $6,8 \%$ & $7,6 \%$ & - & - \\
\hline Sogecable & $7,8 \%$ & - & - & - \\
\hline Vocento & $3,4 \%$ & $4,2 \%$ & $4,4 \%$ & $4,4 \%$ \\
\hline Unidad editorial & $1 \%$ & $1,6 \%$ & $3,2 \%$ & $3,5 \%$ \\
\hline
\end{tabular}

Tabla 2. Cuota de pantalla agregada de los canales en función de los grupos mediáticos (20102013). Fuente: Barlovento Comunicación. Elaboración propia. 
La ampliación de la oferta audiovisual significó, en términos generales, un descenso generalizado de las cuotas de audiencia de todos los canales como consecuencia del reparto de la audiencia. Además, las nuevas cadenas iniciaron su andadura con índices mínimos de audiencia, por ejemplo Boing $(0,7 \%)$ o Nitro $(0,4 \%)$. Algunos canales sí se han hecho un hueco entre las preferencias del telespectador español aunque las cuotas de audiencia siguen siendo residuales (véase el caso de Divinity o de Xplora, cuya cuota media del año 2013 fue de 1,7\%).

En relación al reparto de la audiencia por grupos mediáticos, en el año 2010 CRTVE ejerció de líder concentrando al $24,1 \%$ de la audiencia. Cabe destacar la pérdida gradual de cuota de pantalla de CRTVE desde el año 2010. De ser el grupo líder ha pasado a ocupar el tercer lugar en el año 2013, perdiendo más de siete puntos.

En el año 2011, CRTVE perdió su liderazgo a favor de Mediaset España. Este grupo mediático, como consecuencia de la compra de la televisión en abierto del grupo Prisa, congregó al 26,4\% de la audiencia. Mediaset ostentó ese liderazgo durante tres de los cuatros años estudiados (2011, 2012 y 2013), aunque progresivamente vio reducida su ventaja porcentual con su principal competidor, Atresmedia. Tal como se recoge en la tabla 2, Atresmedia fue afianzando su posición hasta prácticamente alcanzar los resultados de Mediaset, quedándose únicamente a dos décimas de diferencia en el año 2013.

En definitiva, el proceso de fragmentación de las audiencias iniciado con la implantación de la TDT ha derivado en una situación de concentración de la audiencia originada por la reconfiguración de dos grupos mediáticos. La polarización de la audiencia se afianzó en el año 2012, cuando Atresmedia y Mediaset España agruparon a más del 50\% de la audiencia y se consolidó en el año 2013 cuando entre ambos operadores concentraron casi al $60 \%$.

La pugna por el liderazgo entre Atresmedia y Mediaset España continúa durante el año 2014 y se verá afectada por la ejecución de la sentencia del Tribunal Supremo sobre la anulación de nueve canales de la TDT (tres de Atresmedia y dos de Mediaset España, NET TV y VEO TV) que traerá consigo nuevos interrogantes y una nueva reconfiguración del escenario televisivo.

Esta situación de polarización de la audiencia tiene su reflejo en la inversión publicitaria, como estudiaremos a continuación.

\subsection{Inversión publicitaria}

Desde el año 2010, el mercado publicitario español está sufriendo una serie de cambios que han influido considerablemente en su actual configuración. En primer lugar, la crisis económica global que ha afectado drásticamente a las cifras globales de inversión publicitaria. Desde el año 2007 hasta el 2012, ha perdido la mitad de su facturación (53,5\%) (Noticias de la Comunicación, 2013). La recuperación del mercado publicitario va a ser lenta pero las previsiones realizadas hasta el momento tienden a ser positivas para el año 2014.

En segundo lugar, la eliminación de la publicidad en la CRTVE supuso un nuevo reparto de la tarta publicitaria. Y, en tercer y último lugar, las operaciones de fusión y absorción comentadas anteriormente han derivado en una clara situación de duopolio, como veremos a continuación. 


\begin{tabular}{|c|c|c|c|c|c|c|c|c|}
\hline & \multicolumn{2}{|c|}{2010} & \multicolumn{2}{|c|}{2011} & \multicolumn{2}{|c|}{2012} & \multicolumn{2}{|c|}{2013} \\
\hline & Euros & Cuota & Euros & Cuota & Euros & Cuota & Euros & Cuota \\
\hline $\begin{array}{c}\text { Grupo } \\
\text { Atresmedia }\end{array}$ & 690,2 & 27,9 & 681,5 & 30,5 & 639,0 & 35,2 & 725 & 42,6 \\
\hline $\begin{array}{c}\text { Grupo } \\
\text { Mediaset } \\
\text { España }\end{array}$ & 792,9 & 32,1 & 975,0 & 43,6 & 821,5 & 45,3 & 745 & 43,7 \\
\hline $\begin{array}{c}\text { Grupo La } \\
\text { Sexta }\end{array}$ & 276,5 & 11,2 & 255,0 & 11,4 & $\begin{array}{l}115,0 \\
\text { (Ene.- } \\
\text { Sept.) }\end{array}$ & 6,3 & - & - \\
\hline $\begin{array}{c}\text { Cuatro } \\
\text { (Sogecable) }\end{array}$ & 295,1 & 11,9 & - & - & - & - & - & - \\
\hline $\begin{array}{l}\text { Disney } \\
\text { Channel }\end{array}$ & 28,5 & 1,2 & 29,0 & 1,3 & 26,3 & 1,4 & 24,9 & 1,5 \\
\hline $\begin{array}{c}\text { Resto TV } \\
\text { nacionales en } \\
\text { abierto }\end{array}$ & 45,7 & 1,9 & 36,5 & 1,7 & 42,1 & 2,3 & 43,2 & 2,5 \\
\hline
\end{tabular}

Tabla 3. Evolución de ingresos publicitarios y cuota por grupos mediáticos expresado en millones de euros. Fuente: Infoadex. Elaboración propia.

En el año 2010, tras la eliminación de la publicidad comercial de Televisión Española, los operadores privadores se vieron beneficiados por el incremento de los ingresos publicitarios. Mediaset España registraba la cuota de inversión publicitaria más alta del mercado (32,1\%). Al año siguiente, tras la fusión con Cuatro, se incrementó esta cuota hasta alcanzar el 43,6\%, afianzando por tanto su posición de líder en el mercado. Mediaset España mantuvo dicho liderazgo durante los cuatro años analizados, sin embargo, Atresmedia consiguió ir acortando distancias hasta situarse prácticamente en los mismos niveles de facturación. Tras la absorción de La Sexta, Atresmedia consolidó su posición en el mercado publicitario y consiguió mejorar su facturación publicitaria situándose muy próxima a la cuota obtenida por Mediaset.

Cabe destacar que los altos niveles de concentración publicitaria en manos de Atresmedia y de Mediaset han ido incrementándose durante al período estudiado hasta derivar en un sólido duopolio. En la actualidad, dos grupos mediáticos reúnen el 95\% de la inversión publicitaria televisiva en España.

Esta situación ha sido denunciada por la Asociación Española de Anunciantes en varias ocasiones, quienes abogan por "el retorno de la publicidad de una manera parcial que en tiempos de ocupación no supusiera más de lo destinado actualmente a las autopromociones de la propia cadena" (Asociación Española de Anunciantes, 2014: web). Consideran que la vuelta de la publicidad a TVE "sería la solución necesaria a la situación actual y evitaría, en parte, que se estrechara el cerco, agobiante y constante, a la competencia en el sector televisivo" (Asociación Española de Anunciantes, 2014: web).

En definitiva, y a la vista de los datos presentados, el mercado publicitario español es un duopolio en manos de Atresmedia y Mediaset España quienes ostentan la posición de líderes con un dominio claro y absoluto de la inversión publicitaria. 


\section{Pluralismo interno: resultados}

En el año 2012, los resultados del análisis de contenido destacan que existe una importante similitud en los criterios de relevancia de la información de los informativos de noche entre TVE1, A3 e Intereconomía (Tabla 4). En primer lugar, podemos observar que entre La 1 e Intereconomía hay un índice de correlación de 0,87. En segundo lugar se produce una gran semejanza entre A3 e Intereconomía con un índice de correlación de 0,82 . Y, en tercer lugar, a solo una décima del segundo, está la afinidad entre A3 y TVE1, con 0,81 de índice de correlación. Los resultados anteriores muestran un bloque formado por TVE1, A3 e Intereconomía con criterios bastante semejantes para dar relevancia a las noticias. Al margen del tratamiento periodístico que los diferentes informativos conceden a los temas, existe una alta coincidencia en los criterios de selección de la información y su priorización.

\begin{tabular}{cccccccccccccc} 
& \multicolumn{2}{c}{ T5 } & \multicolumn{2}{c}{ A3 } & \multicolumn{2}{c}{ La 1 } & \multicolumn{2}{c}{ La Sexta } & \multicolumn{2}{c}{ Cuatro } & \multicolumn{2}{c}{ Intereconomía } \\
& \multicolumn{2}{c}{ Infor. 21 h. } & \multicolumn{2}{c}{ Noticias 2 } & \multicolumn{2}{c}{ TD 21h. } & \multicolumn{2}{c}{ Noticias 2 } & \multicolumn{2}{c}{ Noticias 20 h. } & \multicolumn{2}{c}{ E1 Telediario } \\
& 2012 & 2013 & 2012 & 2013 & 2012 & 2013 & 2012 & 2013 & 2012 & 2013 & 2012 & 2013 \\
\hline \multirow{2}{*}{ T5 } & - & - & 0,58 & 0,43 & 0,39 & 0,53 & 0,52 & 0,44 & 0,72 & 0,39 & 0,51 & 0,35 \\
A3 & 0,58 & 0,43 & - & - & 0,81 & 0,74 & 0,48 & 0,45 & 0,51 & 0,54 & 0,82 & 0,75 \\
La 1 & 0,39 & 0,53 & 0,81 & 0,74 & - & - & 0,27 & 0,46 & 0,46 & 0,40 & 0,87 & 0,59 \\
Sexta & 0,52 & 0,44 & 0,48 & 0,45 & 0,27 & 0,46 & - & - & 0,45 & 0,45 & 0,31 & 0,51 \\
Cuatr & 0,72 & 0,39 & 0,51 & 0,54 & 0,46 & 0,40 & 0,45 & 0,45 & - & - & 0,53 & 0,17 \\
\multirow{2}{*}{ Inter. } & 0,51 & 0,35 & 0,82 & 0,75 & 0,87 & 0,59 & 0,31 & 0,51 & 0,53 & 0,17 & - & - \\
\hline
\end{tabular}

Tabla 4. Indices de correlación entre las cadenas según orden de relevancia de los titulares (2012-2013). Fuente: Elaboración propia

En este mismo año 2012, el menor índice de correlación entre cadenas se dio entre La 1 y La Sexta con tan sólo un 0,27. El penúltimo valor, de 0,31, fue entre la Sexta e Intereconomía. Por último, la correlación entre La 1 y T5 fue de 0,39 , que ocupa la tercera posición en menor coincidencia. El resto de resultados de la correlación de relevancia de los titulares entre pares de cadenas está en el rango de 0,72 , correspondiente al par Telecinco y Cuatro, y 0,45, que se dio entre La Sexta y Cuatro.

Los resultados para el año 2013 muestran una mayor homogeneidad entre los pares de cadenas. La mayor semejanza se da entre Intereconomía y A3 con un 0,75 , seguido a muy corta distancia por la de La 1 y A3, con un 0,74. En relación al año 2012 se perciben dos cambios sustanciales. Por una parte, desaparece la extraordinaria similitud entre La 1 e Intereconomía que en 2012 fue de 0,87 y en 2013 pasa a un 0,59. Por otra, se pone de manifiesto un fuerte contraste entre Cuatro e Intereconomía, que pasan de un 0,53 en 2012 a un mínimo 0,17 en 2013. El resto de resultados de la correlación de relevancia de las noticias entre pares de cadenas está en el rango de 0,72 , correspondiente al par T5 y Cuatro, y 0,45 que se dio entre La Sexta y Cuatro.

De la evolución de los índices de correlación entre las distintas funciones de relevancia de las cadenas, de 2012 a 2013, se han extraído los siguientes resultados: a) Telecinco aumenta su similitud con TVE1 y la disminuye con el resto de cadenas; b) 
Antena 3 disminuye con todas excepto con Cuatro, con la que aumenta muy levemente; c) La 1 disminuye su correlación muy significativamente con Intereconomía y levemente con Cuatro y Antena 3, en cambio aumenta de forma significativa con La Sexta y Telecinco; d) La Sexta deja de ser antítesis de Intereconomía y La 1 y disminuye la correlación con Telecinco y Antena 3; e) Cuatro disminuye significativamente su similitud con Telecinco e Intereconomía, manteniendo o aumentando muy ligeramente con el resto; f) Intereconomía disminuye en relación a todas las cadenas, especialmente con La 1, Cuatro y Telecinco, y aumenta con La Sexta.

Los índices de correlación entre las cadenas han puesto de manifiesto la consonancia o disonancia en lo que respecta a los criterios de selección y ordenación de los titulares. A continuación analizaremos cuáles son las categorías temáticas a las que pertenecen los titulares seleccionados por cada cadena para reflejar también la mayor o menor semejanza en los temas de los titulares.

En 2012, el alto índice de correlación entre La 1, Antena 3 e Intereconomía también se corresponde con una afinidad en la composición por temas de los titulares. Así, si comparamos la distribución del porcentaje de temáticas para todas las cadenas encontramos que el mayor parecido se da entre La 1 e Intereconomía, seguido por el de Antena 3 e Intereconomía, y el de La 1 y A3. Los temas con valores más altos en las tres cadenas, con distintos porcentajes en cada una de ellas, son Crisis económica, Política y Deportes.

El mayor parecido de distribución por temáticas se da entre La Sexta y Cuatro. Cabe destacar, a este respecto, el alto porcentaje de noticias de Sucesos en los titulares de la Sexta $(10,5 \%)$, y en Cuatro $(13,2 \%)$ frente La $1(2,3 \%)$ o Intereconomía $(2,5 \%)$.

\begin{tabular}{|l|c|c|c|c|c|c|}
\hline & $\begin{array}{c}\text { T5 } \\
\text { Inf. 21 h }\end{array}$ & $\begin{array}{c}\text { A3 } \\
\text { Not. 2 }\end{array}$ & $\begin{array}{c}\text { La 1 } \\
\text { TD 21h }\end{array}$ & $\begin{array}{c}\text { La Sexta } \\
\text { Not. 2 }\end{array}$ & $\begin{array}{c}\text { Cuatro } \\
\text { Not. 20h }\end{array}$ & $\begin{array}{c}\text { Interec. } \\
\text { El TD }\end{array}$ \\
\hline Política & 11,8 & 10,5 & 17,4 & 11,8 & 11,8 & 20,0 \\
\hline Crisis económica & 12,9 & 14,5 & 16,3 & 10,5 & 9,2 & 12,5 \\
\hline Economía & 3,5 & 5,3 & 3,5 & 5,3 & 3,9 & 2,5 \\
\hline Reforma laboral & 4,7 & 3,9 & 5,8 & 3,9 & 2,6 & 6,3 \\
\hline Corrupción & 5,9 & 3,9 & 4,7 & 6,6 & 2,6 & 3,8 \\
\hline Caso Nóos & 5,9 & 1,3 & 1,2 & 6,6 & 2,6 & 5,0 \\
\hline Judicial & 4,7 & 6,6 & 3,5 & 5,3 & 3,9 & 3,8 \\
\hline Intermacional & 4,7 & 5,3 & 10,5 & 3,9 & 7,9 & 8,8 \\
\hline Intermacional sucesos & 1,2 & 2,6 & 2,3 & 1,3 & 2,6 & - \\
\hline Conflictos armados & 1,2 & 1,3 & 1,2 & - & 2,6 & 2,5 \\
\hline Sociedad & 8,2 & 6,6 & 3,5 & 3,9 & 7,9 & 5,0 \\
\hline Manifestaciones/protestas & 9,4 & 3,9 & 3,5 & 9,2 & 6,6 & 5,0 \\
\hline Iglesia católica & - & - & - & 1,3 & 1,3 & - \\
\hline Salud & - & - & 1,2 & 1,3 & - & - \\
\hline Sucesos & 8,2 & 6,6 & 2,3 & 10,5 & 13,2 & 2,5 \\
\hline Meteorología & 3,5 & - & 3,5 & 3,9 & 2,6 & 1,3 \\
\hline Cultura & 3,5 & 3,9 & 4,7 & 1,3 & 2,6 & - \\
\hline Deportes & 5,9 & 19,7 & 15,1 & 9,2 & 13,2 & 18,8 \\
\hline Otros & 4,7 & 3,9 & - & 3,9 & 2,6 & 2,6 \\
\hline N=479 & 85 & 76 & 86 & 76 & 76 & 80 \\
\hline
\end{tabular}

Tabla 5. Distribución de los temas en los titulares por cadenas en 2012 (\%).

Fuente: Elaboración propia

El índice de menor correlación entre cadenas, que se dio entre La 1 y La Sexta, se corresponde con la mayor diferencia en la composición por temáticas de los titulares, 
que no es muy significativa. Así, los bloques de Política, Crisis económica, Economía y Reforma laboral en TVE constituyen el 43,1\% del total de temáticas de los titulares, frente al 31,5\% que representan en La Sexta.

En 2013 existe bastante semejanza en la distribución de temáticas (Tabla 6). Así, la coincidencia más alta es entre Intereconomía y Antena 3, seguidas a escasa distancia por La Sexta y Telecinco, Cuatro y Telecinco, y Cuatro y La Sexta. Cabe destacar un descenso significativo de la coincidencia de distribución temática entre La 1 e Intereconomía, aunque la desemejanza más destacada es entre Cuatro y La 1, que también desciende significativamente respecto a 2012.

\begin{tabular}{|l|c|c|c|c|c|c|}
\hline & $\begin{array}{c}\text { T5 } \\
\text { In. 21 h. }\end{array}$ & $\begin{array}{c}\text { A3 } \\
\text { Not. 2 }\end{array}$ & $\begin{array}{c}\text { La 1 } \\
\text { TD 21h }\end{array}$ & $\begin{array}{c}\text { La } \\
\text { Sexta } \\
\text { Not. 2 }\end{array}$ & $\begin{array}{c}\text { Cuatro } \\
\text { Not 20h }\end{array}$ & $\begin{array}{c}\text { Interec. } \\
\text { El TD }\end{array}$ \\
\hline Política & 15,5 & 17,4 & 16,4 & 16,7 & 10,8 & 15,7 \\
\hline Crisis económica & 4,8 & 5,8 & 6,8 & 2,6 & 4,1 & 5,7 \\
\hline Economía & 6,0 & 7,2 & 8,2 & 6,4 & 2,7 & 7,1 \\
\hline Reformalaboral & - & - & - & - & - & - \\
\hline Comupción & 14,3 & 8,7 & 6,8 & 15,4 & 12,2 & 14,3 \\
\hline Caso Nóos & 13,1 & 10,1 & 6,8 & 9,0 & 12,2 & 11,4 \\
\hline Judicial & 3,6 & - & - & 1,3 & 1,4 & 1,4 \\
\hline Intemacional & 6,0 & 7,2 & 8,2 & 5,1 & 5,4 & 8,6 \\
\hline Intemacional sucesos & 3,6 & 2,9 & 4,1 & 1,3 & 2,7 & 2,9 \\
\hline Conflictos armados & 1,2 & 1,4 & - & - & 2,7 & - \\
\hline Sociedad & 6,0 & 5,8 & 4,1 & 7,7 & 9,5 & 4,3 \\
\hline Manifestaciones/protestas & 7,1 & 2,9 & 6,8 & 7,7 & 6,8 & 4,3 \\
\hline Iglesia católica & 2,4 & 2,9 & 4,1 & 2,6 & 2,7 & 2,9 \\
\hline Salud & 1,2 & 4,3 & 5,5 & 3,8 & 4,1 & 2,9 \\
\hline Sucesos & 8,3 & 8,7 & 2,7 & 11,5 & 9,5 & 7,1 \\
\hline Meteorología & 2,4 & -- & 1,4 & 2,6 & 5,4 & - \\
\hline Cultura & 2,4 & 1,4 & 5,5 & 2,6 & 5,4 & 1,4 \\
\hline Deportes & 1,2 & 11,6 & 11,0 & 3,8 & 1,4 & 10,0 \\
\hline Otros & 1,2 & 1,4 & 1,4 & - & 1,4 & - \\
\hline N=448 & 84 & 69 & 73 & 78 & 74 & 70 \\
\hline
\end{tabular}

Tabla 6. Distribución de los temas en los titulares por cadenas en 2013 (\%).

Fuente: Elaboración propia

En cuanto a la evolución de las temáticas de los titulares en las distintas cadenas, de 2012 a 2013, se han extraído los siguientes resultados: a) Telecinco aumenta significativamente su coincidencia con Intereconomía y Cuatro, y, en menor medida, con Antena 3 y La Sexta; en cambio disminuye con La 1; b) Antena 3 aumenta significativamente su coincidencia con La Sexta e Intereconomía y, muy ligeramente, con respecto a $\mathrm{La}$ 1, en cambio disminuye fuertemente con Cuatro; c) La 1 disminuye fuertemente su coincidencia con Cuatro y, en menor medida con Intereconomía; d) La Sexta aumenta su coincidencia con Intereconomía y, en menor medida, con Cuatro; e) Cuatro disminuye fuertemente en relación a Intereconomía.

Los resultados del análisis de contenido sobre los titulares en los programas seleccionados confirman la segunda hipótesis que plantea que el pluralismo interno informativo se ha reducido debido a la lógica de comercialización de la información diaria, aunque con matices en cada cadena. Los resultados para el año 2013 muestran una ligera tendencia a la homogeneidad entre los pares de cadenas en lo que respecta a los criterios de relevancia de los titulares. Igualmente, en este mismo año, se ha evolucionado a una mayor semejanza en la distribución de temáticas dentro de los titulares. 
A grandes rasgos, los resultados muestran que el informativo de noche de La 1 conserva en líneas generales las características principales de la información en una televisión pública: un fuerte componente de noticias políticas, económicas y de ámbito internacional, con pocas concesiones a las noticias sobre temas anecdóticos, pero con una presencia importante de informaciones dedicadas a deportes. Los informativos de las cadenas más comerciales, Telecinco, Antena 3 y Cuatro han tendido a ser más coincidentes con los canales más polarizados políticamente, La Sexta e Intereconomía.

\section{Conclusiones}

En el período 2010-2013, la implantación de la televisión digital terrestre ha provocado cambios importantes en el sistema televisivo español. Estos cambios han afectado de forma contradictoria al pluralismo externo si nos atenemos a los indicadores analizados. Respecto a la propiedad de las compañías con licencia de TDT, ninguno de los socios ha abandonado el negocio de la televisión; las operaciones de compra entre operadores a las que hemos hecho referencia más arriba han provocado que dichos socios hayan cambiado su participación accionarial y su presencia en la gestión de las televisiones, pero no ha llevado a la reducción del número de propietarios significativos presentes en el sector televisivo. Por tanto, observamos que la concentración se ha producido en la gestión de las televisiones y en la capacidad de emisión con cobertura nacional que se encuentran prácticamente en manos de tres empresas, pero no en el número de propietarios de las mismas.

Respecto a la inversión publicitaria, la concentración es altísima, pues hay un predominio claro de Mediaset España y Atresmedia. Respecto a la audiencia, se podría decir que el pluralismo externo se ha ampliado, pues el aumento del número de canales privados ha permitido la presencia de nuevas cadenas, nuevos contenidos y nuevos puntos de vista; el consumo no se ha concentrado sino que se ha dividido -fragmentado- en todos esos canales.

Desde el punto de vista de la información, la fuerte competencia entre los canales de televisión generalistas ha reducido el pluralismo interno por la lógica de comercialización de la información. La tendencia a la comercialización ha provocado una mayor homogeneidad en el conjunto de las noticias comunes, es decir, con un claro interés público. La fuerte competencia entre canales por conseguir audiencias amplias, en un proceso claro de fragmentación de las mismas por el mayor número de canales, ha propendido a introducir mayores recursos de entretenimiento en las noticias. Estos recursos pueden apelar tanto a la espectacularización de las noticias duras, relativas a la política, corrupción, la contestación social frente a la crisis, etc., como a la inserción de un mayor número de noticias blandas que tienen que ver más con el entretenimiento que con la información sobre los asuntos de interés público.

No se puede concluir que haya una relación determinante entre el grado de pluralismo externo e interno en lo que respecta a la información. Más bien los factores determinantes del pluralismo en este período han sido las diferentes estrategias empresariales que pugnan por delimitar productos informativos adecuados a las exigencias de diferentes tipos de públicos. Es decir, aún cuando el pluralismo externo se ha ampliado desde el punto de vista de la audiencia, el pluralismo interno continúa siendo deficitario en la cultura informativa española. 


\section{Referencias bibliográficas}

ASOCIACIÓN ESPAÑOLA DE ANUNCIANTES (28 de marzo de 2014): "Comunicado de la aea ante la Comisión de Control Parlamentario del pasado jueves", en: http://www.anunciantes.com/comunicacion_ficha.php?s=21\&c=357 [Consulta: 8 de abril de 2014]

BARLOVENTO COMUNICACIÓN: "Informe Audiencias televisivas (2010, 2011, 2012, 2013)", en: http://www.barloventocomunicacion.es/publicaciones.html [Consulta: 5 de abril de 2014]

BUSTAMANTE, Enrique (2008): "Modelos internacionales de TDT: La política y los lobbies pueden frustrar las expectativas de diversidad". Diálogos de la comunicación. Revista académica de la Federación Latinoamericana de Facultades de Comunicación Social, $\mathrm{n}^{\mathrm{o}}$ 77, julio-diciembre, pp. 1-23.

BUSTAMANTE, Enrique y CORREDOR, Patricia (2012): "La política audiovisual de los gobiernos de Zapatero. Balance de la Segunda Legislatura", en LAMUEDRA GRAVAN, María (coordinadora): El futuro de la televisión pública. La necesaria alianza con la ciudadanía. Madrid, Editorial Popular, pp. 61-86.

COMUNICACIÓN PODER JUDICIAL (19 de diciembre de 2013): "El Tribunal Supremo ordena cesar las emisiones de nueve canales de Televisión Digital Terrestre adjudicados sin concurso previo", en: http://www.poderjudicial.es/cgpj/es /Poder_Judicial/Tribunal_Supremo/Sala_de_prensa/Documentos_de_Interes/El_Tr ibunal_Supremo_ordena_cesar_las_emisiones_de_nueve_canales_de_Television Digital Terrestre adjudicados $\overline{\text { sin }}$ concurso previo [Consulta: 11 de abril de $2 \overline{0} 14]$

CONSEJO DE EUROPA (1994): “4ème Conférence ministérielle européenne sur la politique des communications de masse. Les media dans une société démocratique". Praga, 7-8 de diciembre de 1994. Rapport d'activité du Comité d'experts sur les concentrations des media et le pluralisme. MCM (94) 5". Estrasburgo, p. 8.

DÍAZ-GONZÁLEZ, María-Jesús y LABIO BERNAL, Aurora (2012): "Balance del primer año de Telecinco como mayor empresa privada de televisión en España: impacto sobre el pluralismo". Congreso Internacional Comunicación y Riesgo, Asociación Española de Investigación en Comunicación, Tarragona, en: http://www.ae-ic.org/tarragona2012/contents/esp/abstracts395fl.html?id_seccion= 3\&id_slot=25 [Consulta: 5 de noviembre de 2013]

DÍAZ-GONZÁLEZ, María-Jesús y QUINTAS FROUFE, Natalia (2013): “La absorción de La Sexta por Antena 3 de Televisión y sus efectos en el pluralismo externo del sistema televisivo español". Observatorio (OBS) Journal, vol. 7, n ${ }^{\circ}$. Lisboa, OberCom, pp. 63-90.

ENTMAN, Robert M. (1989): Democracy Without Citizens. Media and the Decay of American Politics. Oxford: Oxford University Press.

GARCÍA-SANTAMARÍA, José V. (2013): “Televisión y concentración en España: el duopolio de Mediaset y Atresmedia". Palabra clave, vol. 16, $\mathrm{n}^{\circ}$ 2, Cundinamarca, Colombia, Universidad de La Sabana, pp. 366-397. 
HALLIN, Daniel C. y MANCINI, Paolo (2004): Comparing media systems: Three models of media and politics. Cambridge, Cambridge University Press.

HUMANES, María Luisa; MONTERO, María Dolores; MOLINA, Ramón; y LÓPEZ BERINI, Antonio (2013): "Pluralismo y paralelismo político en la información televisiva en España". Revista Latina de Comunicación Social, $\mathrm{n}^{\circ}$ 68. La Laguna (Tenerife), Universidad de La Laguna, pp. 566-581. http://dx.doi.org /10.4185/RLCS-2013-990

IOSIFIDIS, Petros (2010): "Pluralism and Concentration of Media Ownership: Measurement Issues". Javnost-The public, vol. 17, n 3. Ljubljana, Slovenia, European Institute for Communication and Culture, pp. 5-22.

JUST, Natascha (2009): "Measuring media concentration and diversity: new approaches and instruments in Europe and the US". Media,Culture \& Society, vol. 31, $\mathrm{n}^{\circ}$ 1. Sage Publications, pp. 97-117. http://dx.doi.org/10.1177/0163443708098248

KARPPINEN, Kari (2013): Rethinking Media Pluralism. New York, Fordham University Press.

K. U. LEUVEN-ICRI, JÖNKÖPING INTERNATIONAL BUSINESS SCHOOL MMTC, CENTRAL EUROPEAN UNIVERSITY - CCMS, ERNST \& YOUNG CONSULTANCY BELGIUM (2009): Independent Study on indicators for Media Pluralism in the Member States - Towards a Risk-Based Approach. Prepared for the European Commission. Directorate-general Information Society and Media. Leuven, julio, en: https://ec.europa.eu/digital-agenda/sites/digital-agenda/files/final_report_09.pdf [fecha de consulta: 9 de mayo de 2014]

LEÓN GROSS, Teodoro (2006): "La agenda de los noticiarios", en DÍAZ NOSTY, Bernardo (dir.): Tendencias'06. Medios de Comunicación. El año de la televisión. Madrid, Fundación Telefónica, pp. 271-279.

LÓPEZ, José Antonio y CUENCA, Francisco (2005): “Televisión e información: análisis de los criterios de televisión de calidad en los informativos de las cadenas nacionales". Comunicar, $\mathrm{n}^{\circ} 25$. Huelva, Grupo Comunicar.

MIGUEL, Juan Carlos y POZAS, Víctor (2009): “¿Polarización ideológica o económica? Relaciones entre los medios y el poder político y corporativo". Viento Sur, $n^{\circ}$ 103. Madrid, Viento Sur, pp. 43-52.

MILBRATH, Lester W. y GOEL, M. Lal (1977): Political Participation. How and Why Do People Get Involved in Politics? Lanham, University Press of America, $2^{\mathrm{a}}$ ed. Edición original: 1965.

MONTERO, María Dolores y HUMANES, María Luisa (2013): "El pluralismo informativo en la televisión española: entre la comercialización y la polarización política”. XIII Congreso Internacional IBERCOM. Comunicación, Cultura y esferas de poder, Santiago de Compostela.

NORDENSTRENG, Kaarle (2006): “Four Theories of the Press' reconsidered”, en CARPENTIER, Nico; PRUULMANN-VENGERFELDT, Pille; NORDENSTRENG, Kaarle; HARTMANN, Maren; VIHALEMM, Peeter y CAMMAERTS, 
Bart (eds.): Researching media, democracy and participation: The intellectual work of the 2006 European Media and Communication Doctoral Summer School. Tartu, Tartu University Press, pp. 35-45.

NOTICIAS DE LA COMUNICACIÓN (2013): "La inversión publicitaria baja un $14,3 \%$ hasta junio y un $53,5 \%$ desde 2007 '. Noticias de la Comunicación, $\mathrm{n}^{\circ} 338$. Madrid, pp. 50-51.

STRÖMBÄCK, Jesper (2005): "In Search of a Standard: four models of democracy and their normative implications for journalism". Journalism Studies, vol. 6, n ${ }^{\circ} 3$. London, Routledge, pp. 331-345. http://dx.doi.org/10.1080/14616700500131950

TRIBUNAL SUPREMO. SALA DE LO CONTENCIOSO (13 de febrero de 2014a): "Auto 1082/2014. Recurso de reposición contra auto de 18-12-2013 en ejecución de sentencia", en: http:/www.poderjudicial.es/search/doAction?action=contentpdf\&databasematch $=$ TS \&reference $=6977882 \& 1$ in $\mathrm{ks}=\&$ opti mize $=20140303 \&$ publicinterface $=$ true [Consulta: 11 de abril de 2014]

TRIBUNAL SUPREMO. SALA DE LO CONTENCIOSO (25 de marzo de 2014b): "Auto 2512/2014. Solicitud de requerimiento a la Administración para que comunique el responsable del cumplimiento de la sentencia y para que decrete el efectivo cese de las emisiones de los canales de televisión", en: http://www.poderjudicial.es/search/doAction?action=contentpdf\&databasematch $=$ TS \&reference $=7014239 \&$ links $=\&$ optimize $=20140407 \&$ publicinterface=true [Consulta: 12 de abril de 2014]

VIKE-FREIBERGA, Vaira; DÄUBLER-GMELIN, Herta; HAMMERSLEY, Ben y POIARES PESSOA MADURO, Luis Miguel (2013): “A free and pluralistic media to sustain European democracy. The Report of the High Level Group on Media Freedom and Pluralism", en: https://ec.europa.eu/digital-agenda/sites/digitalagenda/files/HLG\%20Final\%20Report.pdf [Consulta: 9 de mayo de 2014]

ZALLO, Ramón (2010): La política de Comunicación Audiovisual del gobierno socialista (2004-2009): un giro neoliberal”. Revista Latina de Comunicación Social, $\mathrm{n}^{\circ}$ 65, La Laguna (Tenerife), Universidad de La Laguna, pp. 14-29. http://dx.doi.org/10.4185/RLCS-65-2010-880-014-029 